\title{
STUDY OF THE PARAMETERS TO MITGATE THE MANUAL TRANSMISSION RATTLE NOISE FOR PASSANGER CARS.
}

\author{
Felipe Hajime Ohira, Leandro Peres Oliveira, Marcio Caçula, Massimiliano Corazza \\ General Motors \\ felipe.ohira@gm.com ; leandroperes.oliveira@gm.com; marcio.cacula@gm.com; \\ massimilano.corazza@gm.com
}

\begin{abstract}
SUMMARY
This PAPER has the proposal to study the physics and the propagation of the transmission gear rattle, starting with the engine irregular combustion and the fluctuation of the torque generate the torsional vibration that passes to the transmission and excite the internal components and appears the noise. With simulations we will understand the enablers that act to reduce this torsional vibration and decrease the noise level, measure the hardware and correlate with the simulation to define a robust design that reach the vehicle technical requirements and a good satisfaction to the customer.
\end{abstract}

\section{INTRODUCTION}

Aiming for the Future and to become more competitive in the market, the Companies are always searching for best compromise in terms of energetic efficiency (fuel consumption and emissions) and some new technologies are being applied for the projects. One of these technologies are the engine three Cylinders and manual transmissions six speeds. With this powertrain combination the increase of the transmission gear rattle noise is eminent. One of the challenges of the vehicle development are to mitigate this noise level to deliver the best product to the customer.

The purpose of this PAPER is to study, perform simulation and vehicle measurements the influence of some parameters to mitigate the transmission rattle and set a robust design that reach the vehicle technical requirements and satisfactory to the customer.

\section{Concepts and enablers to mitigate the rattle}

The transmission rattle noise is the metallic noise coming from the transmission components and is qualitatively similar to the marbles in a shaken metallic can. Depending on the level of the noise, the customer could associate to some issue involving the powertrain and in a wrong way.

This phenomenon is generate mainly by the fluctuation of the torque from the engine crankshaft when occurs the cylinder explosions sequences. These irregularities increase the torsional vibration that is transmitting to the transmission input shaft, causing loose gear in transmission to loose contact and appears rattle in lash region according to the backlash defined.

We can define the rattle as a noise of the system and not a specific problem from the teeth of the gears or the transmission, the complexity is extend to the entire powertrain with the non-linear 
elements, and sometimes one noisy manual transmission could be implemented to another powertrain combination and present an acceptable noise level.

Gear rattle is associated with the characteristic structure and airborne noises that are radiated to the environmental by the impact of unselected transmission gear pairs when excited with the torsional vibration.

We could divide the physics of the gear rattle in source, path and receiver, according to the picture:

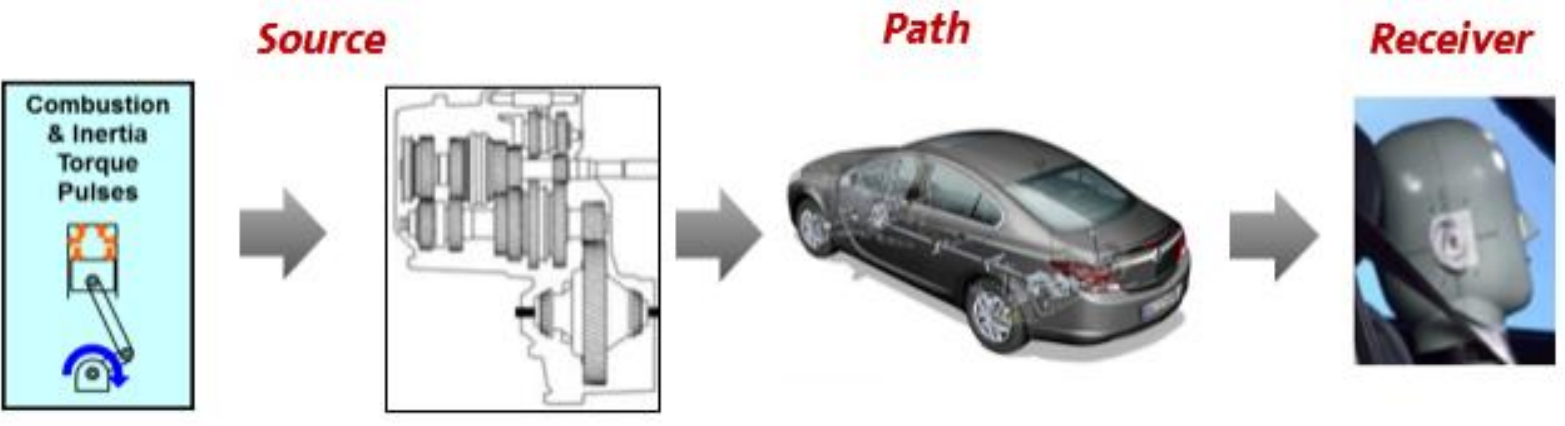

Fig.01: Source, Path and Receiver demonstration.

Multiple solution sets are possible at source and path to meet the acceptable noise and the vehicle requirements, but this should be carefully analyzed as all the enablers have your benefits but also could have a downside as costs and mass increased or loose efficiency.

Source is the propulsion system, it will be divided in engine excitation, and transmission assembly and path is divided in structural borne path and air borne path.

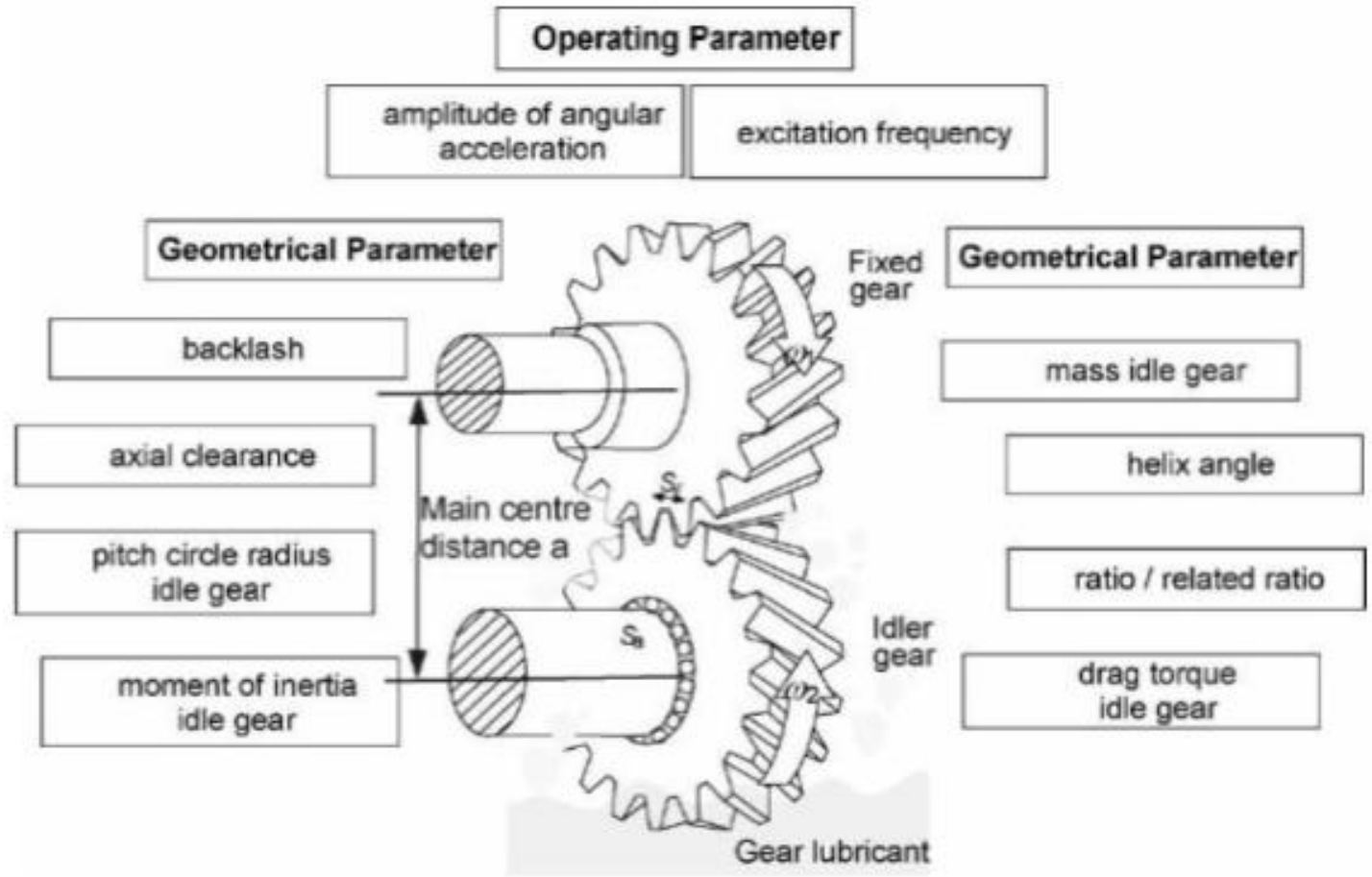

Fig.02: Parameter influence of rattling. 
Engine excitation is related from the combustion and inertia of the components, torque and pulses, powertrain calibration definition, performing the measurements to verify the cycle torque, amplitude, variation, torsional vibration level. To reduce or filtrate the torsional vibration the best enabler is the flywheel per mass, inertia or if it is a critical level, a dual mass flywheel could be applied as a good solution.

Transmission assembly covers the clutch system that is connected to the flywheel and it is the next enabler to be verified and could help to filter and isolate the level of the vibration that continue arriving to input shaft of the transmission and correlates to the transmission rattle sensitivity has a level of noise. Inside the gearbox, the enablers are the drag torque, inertia of the components, gear backlash, housing and the structures.

The chart below resumes all the way, from the engine excitation until the passenger's compartment, and the possible enablers to reduce the rattle noise:

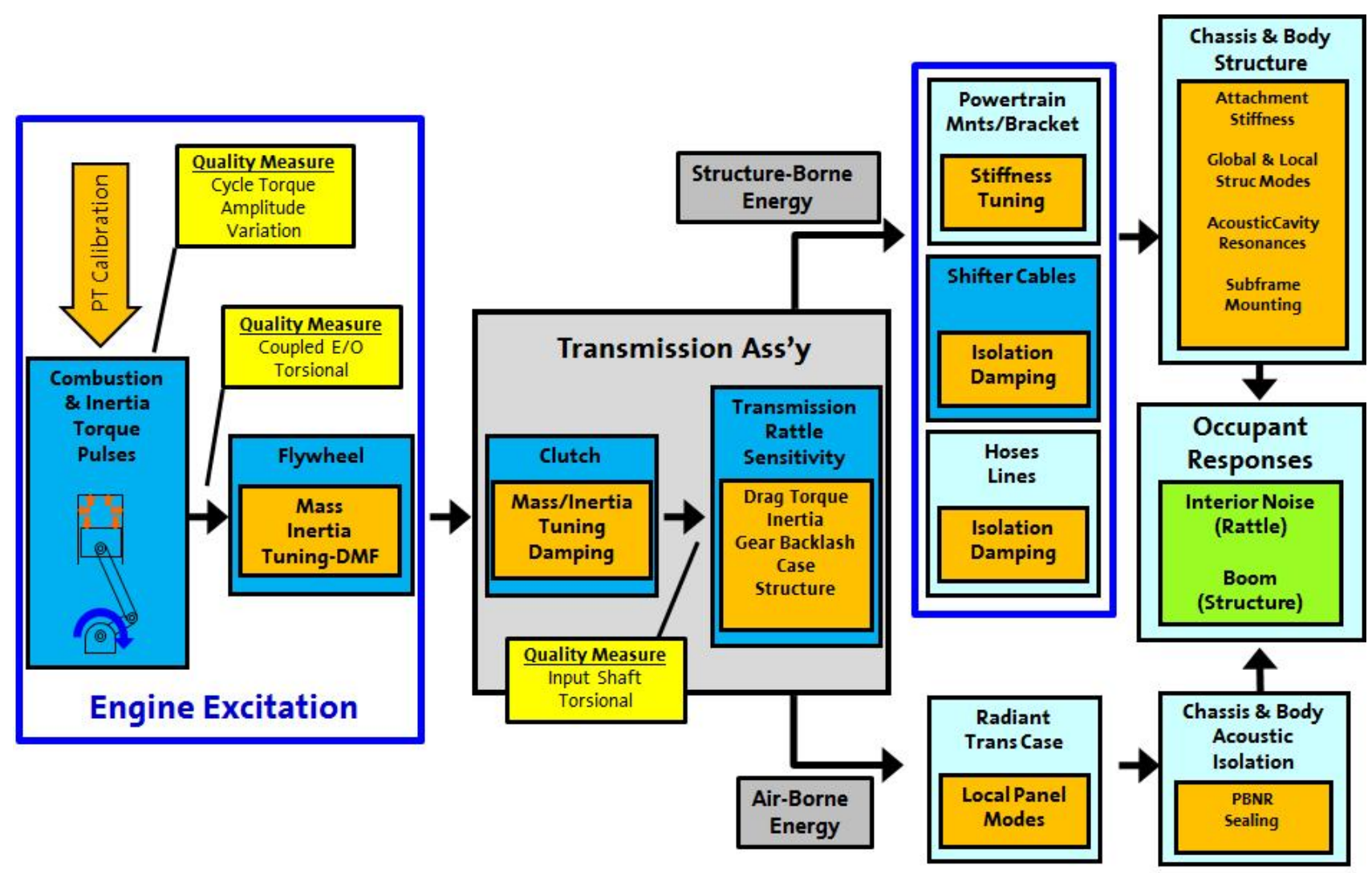

Fig.03: Physics of rattle noise and enablers that influence the noise.

After generate the rattle noise it continues in two forms to the vehicle and the passenger's compartment, structural borne energy and air borne energy.

Structure borne is divided in powertrain mounts and bracket defining the stiffness and tuning of the dampers, external shift cables working on isolation and damping of the connectors on the shifter housing or in the connections on the transmission cover, hoses and clutch release systems 
isolating the vibration with damper. Chassis and body structures as attachments and stiffness of the side shafts, the acoustical cavity, structure modes and subframe should also help to isolate the vibration. After this way, the vibration arrives to the occupant generating the boom on the structure. Air borne is divided in radiant transmission case with local panels and modes around the transmission, chassis and body acoustic isolation as PBNR and sealing. After these isolations, the noise arrives to the interior of the vehicle and to the customer rear.

\begin{tabular}{|l|l|}
\hline \multicolumn{2}{|c|}{ Active measures/reduction of noise generation } \\
\multicolumn{2}{|c|}{ Internal }
\end{tabular}




\subsection{Engine Excitation}

On the engine development always the search for power, efficiency as emissions and fuel consumption are the scope of the projects. Reduction of the cylinders and elimination friction of the components to uses all the energy generated on the combustion are some of the parameters to reach the targets.

With the reduction of the cylinders, the combustion time enlarge the fluctuation of the torque increasing the irregularities, and consequently, a higher torsional vibration is noticed. In addition, the components losing the friction, the system run better and the efficiency increases, but all torsional vibration pass to the crankshaft until the flywheel with less isolation (more friction on the engine, more energy lost, less torsional vibration on the system).

Powertrain calibration map the engine to improve the performance, increase the vehicle drivability, as the response when the vehicle are with some loads. This could be an enabler to also reduce the fluctuation of the torque and torsional vibration, but depending on the case, the engine performance could be limited and the response of the vehicle could not Pleasure the driver, as they would like. For example, limit the torsional vibration on low torque and the vehicle start to have slow recovery after reduction of velocity.

Flywheel absorb the impulses from the crankshaft and it is another enabler to reduce the torsional vibration. As it is connected to the crankshaft, mass and inertia increased, could reduce the torsional vibration that transmitted to the clutch system. Increasing the mass and the inertia, the components, as bushings, seals and crankshaft needed to be reinforced due to the loads increasing. The package inside transmission bell housing could be also a challenge for the project. A bit downside is the reduction of fuel consumption, as you need more energy to rotate a heavy flywheel. On the picture below you can find a measurement with the same vehicle and only variating the flywheel inertia and mass. You can notice from one flywheel to another we can reduce almost $900 \mathrm{rad} / \mathrm{s}^{2}$.

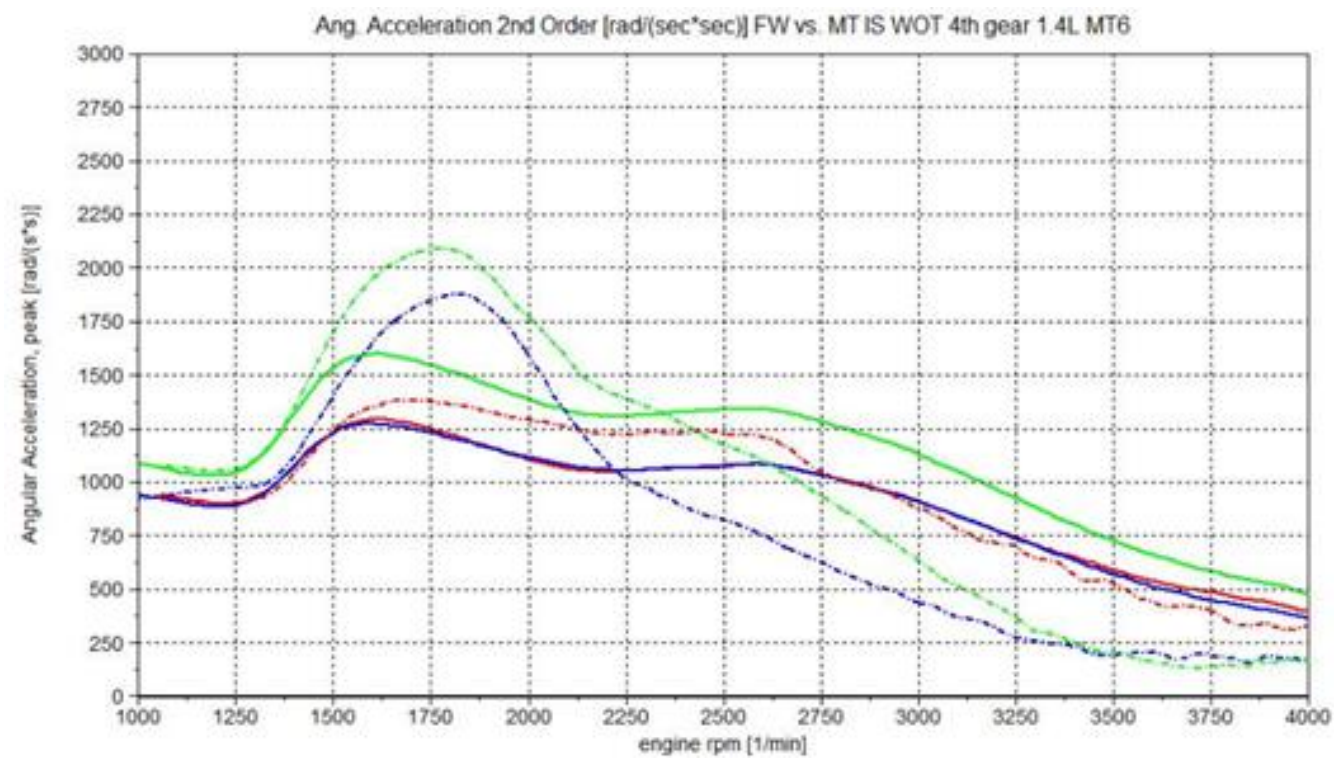

Fig.04: Graph Angular Acceleration vs. engine rpm comparing flywheel mass and inertia influence 
When the torsional vibration is increasing considerable, the best solution is to implement dual mass flywheel on the engine. The DMF has two independent mass, one continue to belong to the engine inertia and the other one, become part of the transmission mass inertia. These two mass are interleafed by a sophisticated system of damper springs arc-shaped, long length, which amounts to almost 360 degrees and is practically immersed in special grease. A bearing allows the movement of the two masses and the result is a large damping capacity of the torsional vibration. The DMF is not a reality for the emergent markets, due to the high costs involved, when compared with the single mass flywheel.

The engine has some good enablers to reduce the level of the torsional vibration that should arrive to the transmission, but some of the downsides needed to be measured as they are reflecting costs, increase of mass/inertia and decreasing the system efficiency.

\subsection{Transmission Assembly}

After the engine combustion and the rotation of the flywheel, the next step is the clutch system that has the responsibility to transmit the torque until the transmission. Also has the function to absorb the irregular vibration coming from the engine with the clutch disc.

Clutch disc has a torsional damped device with some set of springs that act to absorb the vibration and pass a smooth torque until to the transmission input shaft. Depending on the vibration level, the design could increase the complexity and have, for example, eight springs, with two stages on the main damper, where we have a first stage with lower stiffness for better isolation and a second stage increasing the spring stiffness for the torque increase.

The torque and the noise level required are some of the important parameters to consider on the dimension of the clutch system. With the torque, we can define the clamp load necessary to transmit the maximum torque to the transmission. With this clamp load, we check the structural components of the clutch disc and set the spring windows that are possible to implement. In addition, it is defined the disc wind up curve and the minimum stiffness could be implement $\left(\mathrm{Nm} /{ }^{\circ}\right)$.

It was notice, with a lower stiffness and a big angle to the springs act reduce the torsional vibration coming from the engine, but could generate a resonance peak increasing the rattle noise. Sometimes this resonance appears in a short RPM range (for example between 800 to 1000 RPM), the noise happen in a short period, and the customer could not even notice it in some cases. Another parameter of the clutch disc is the hysteresis that are the friction on the clutch disc that elongate or distribute the torsional vibration along the speed increasing. 


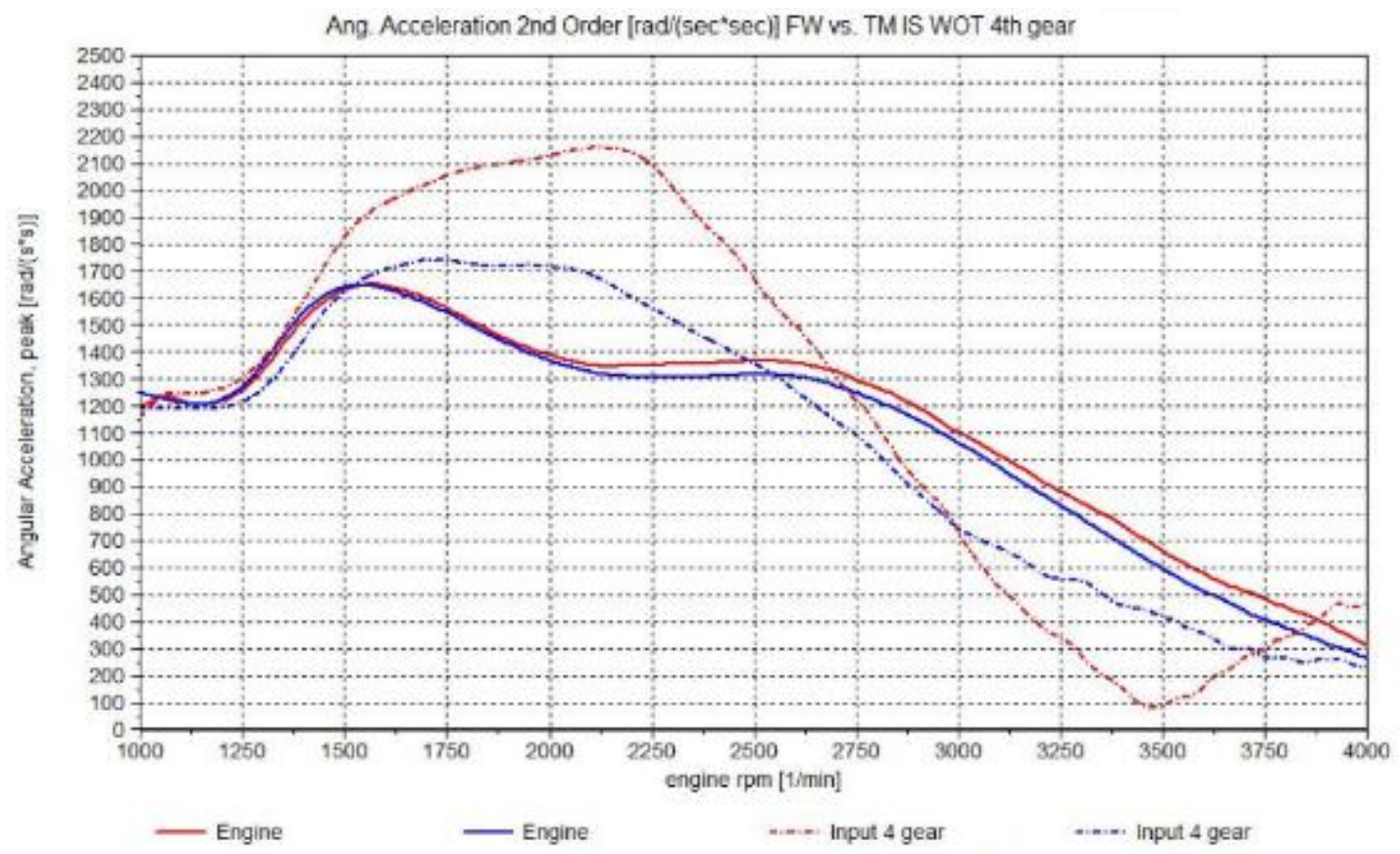

Fig.05: Graph Angular Acceleration vs. engine rpm comparing stiffness and hysteresis of the clutch disc.

Increasing the mass and the inertia of the clutch disc could also contribute to reduce the vibration, but with this, the shift impulse is increased and the synchronizers system inside the transmission needed to be more robust or if the system could not have changes, this could cause a degradation on the shift quality.

Backlash is the technical name given to clearances found between the gear tooth pairs. It occurs due to the dimensional tolerances chosen during the gear manufacturing stage, allowing also a proper lubrication of the contact surfaces. Low values of backlash are desirable, but it cannot be reduced significantly without component wear degradation. This should be carefully analyzed and apply the lowest possible backlash. 


\section{Front and transverse mounted transmission |}

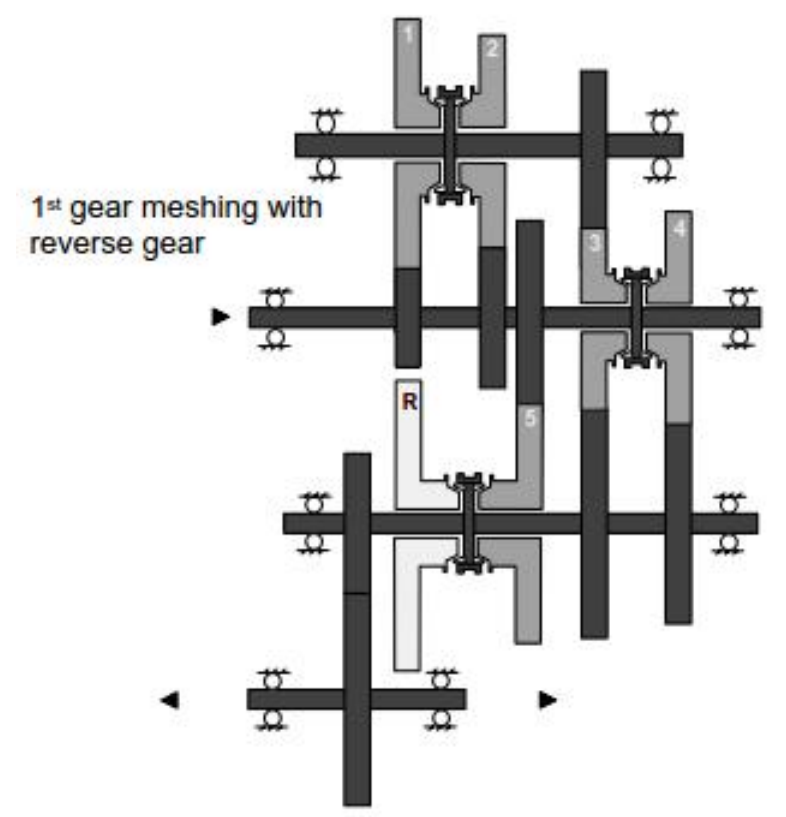

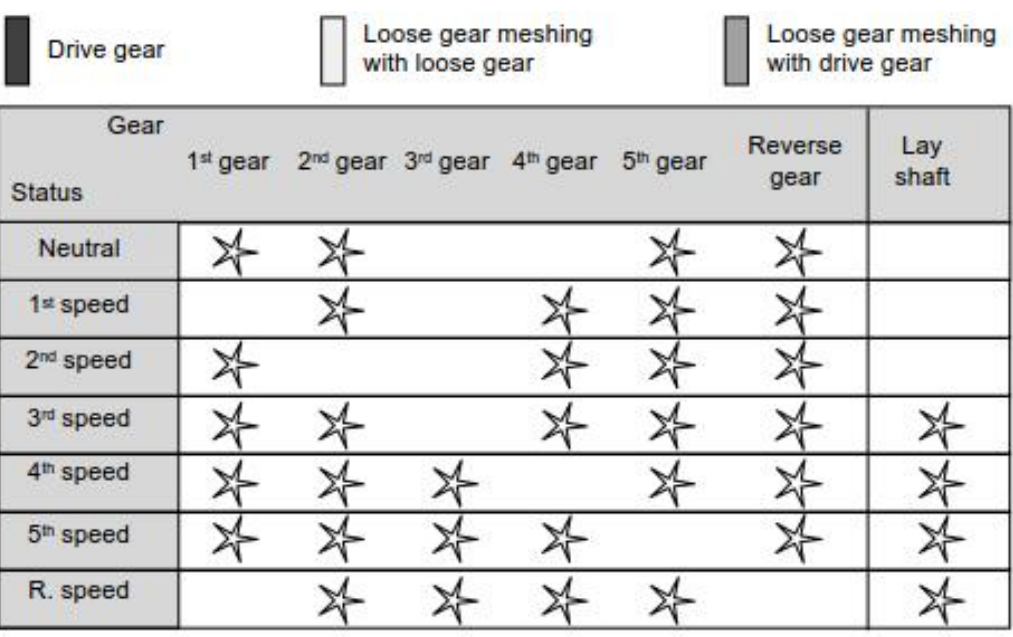

Fig.06: Rattling Meshes in a 5-speed manual front and transverse mounted transmission

Drag torque could be increased by adding a viscosity oil lubricant, but this also interferes to the efficiency of the transmission. In addition, the viscosity could increases on lower temperatures and degradation the shifting quality.

Thickness of the transmission housing or some encapsulation also could contribute to isolate the rattle propagation, but on the measurements and vehicle, evaluations did not present satisfactory results that justify the costs and mass involved on this implementation. In addition, the encapsulation brings some concerns about the heat exchange and the transmission could increases considerable the temperature. 

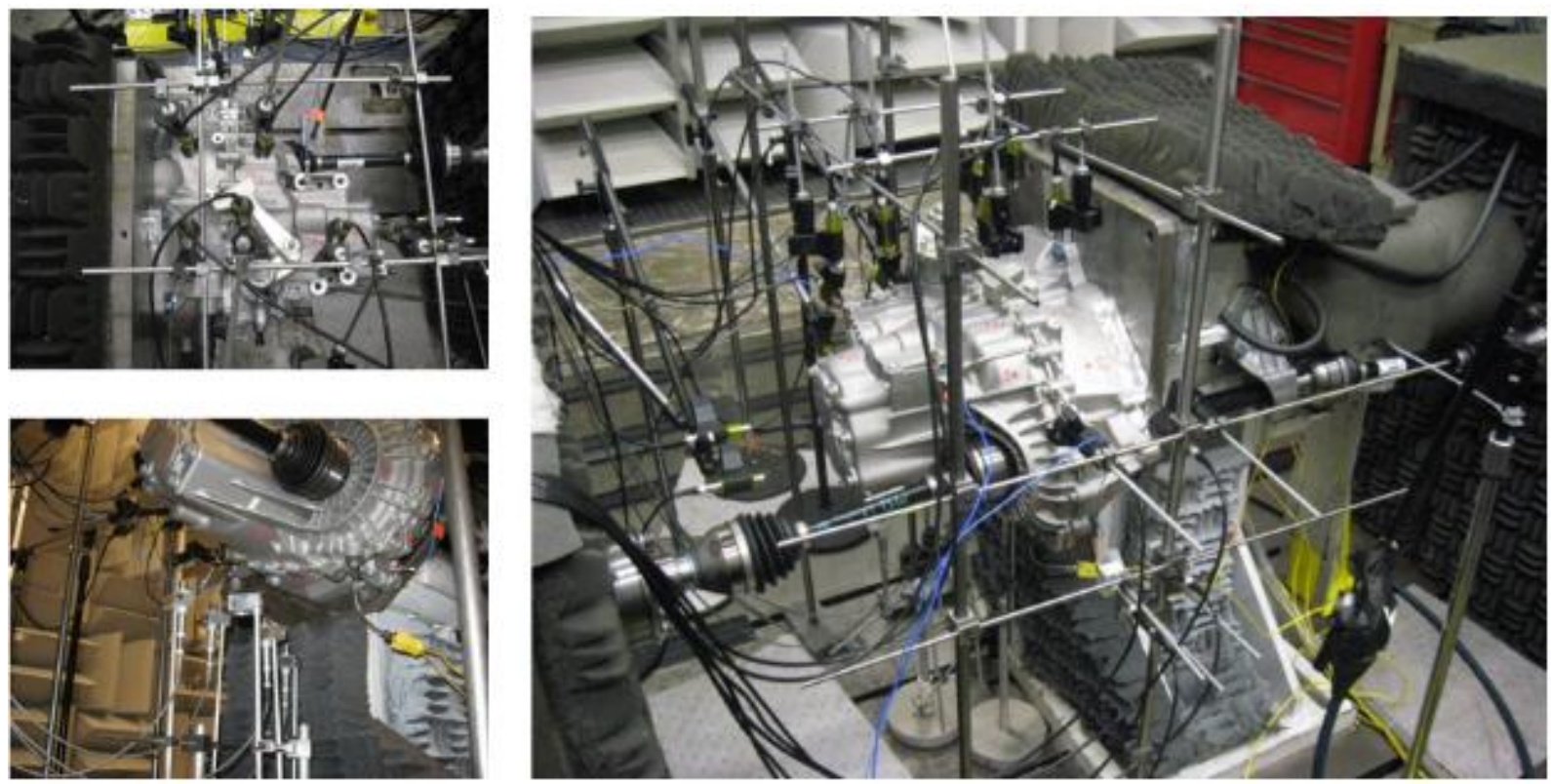

Fig.07: Transmission drag torque measurement

\subsection{Structure borne energy}

After the transmission excitation and generation of the rattle, one of the energy propagation is with the vehicle structure and arrives to the passenger's compartment and for the ears of the customer.

A vehicle's powertrain mounts are its primary line of defense against undesirable noise, vibration, and harshness (NVH) transferred from the engine, transmission, accessory and suspension systems. Therefore, the most important function of a powertrain mounting system is to minimize the energy transfer from the powertrain to the vehicle body. For this reason, mod of the optimization efforts have focused on the 0 to $40 \mathrm{~Hz}$ range for improving the ride and handling and on the 40 to $300 \mathrm{~Hz}$ range for improving the isolation of engine noise. Isolation of the high frequency structure-borne noise, defined as 300 to $1000 \mathrm{~Hz}$.

To meeting the low frequency requirements, an effective design of a mounting system must take into account the interaction of the structural modal characteristics and dynamics of the mount elastomeric component, the brackets, and the supporting structure.

The mount and bracket system for a single mounting location consists in body structure, the body-side bracket, the mount structure, the mount elastomeric component, the mount insert, the powertrain-side bracket, and the powertrain supporting structure. At high frequencies, each of these can play a significant role in the noise transfer.

The role played by the powertrain side structure is to act a support structure for the powertrain-side bracket. This means that it will also define the dynamics which are transmitted to the bracket. Therefore, it is very important that this be a very rigid structure that does not deform readily to the operating forces. The powertrain-side links the powertrain structure to the inner insert of the mount. This is often designed as a function of packaging constraints. The dynamic characteristics of this bracket are very important to 
the high frequency noise path because at bracket resonances there is typically a magnification of the powertrain displacements. The mount is typically a metal core surrounded and bonded to by the elastomeric component of the mount. This is used to secure the mount to the powertrain-side bracket and then the rubber is bonded to the insert. The insert is usually rigidly bolted or tightly fitted to the powertrain bracket and, in fact, becomes part of the bracket for system analysis purposes. In this paper the mount elastomeric component refers to the isolation and damping material enclosed by the mount structure. This can be a rubber, a synthetic rubber compounds. On the other hand, a fluid filled rubber. The fluid filled mounts are implemented to provide increased damping at low frequencies. Each of these types of elastomeric components has its own complex dynamic stiffness functions based on the elastic and damping properties. The body side brackets are the brackets that are welded or bolted to the body and connect the mount structure to the body structure. The mount, which is on the body-side of the elastomeric material, is actually a type of body-side bracket. The body-side brackets, if they exist, and the mount structure, which is bonded to the elastomeric material, should be considered one component for analysis purposes.

The body side structure is the section of the body rail or frame, which supports the powertrain mount and body-side brackets. This structure is often reinforced with extra section thickness etc. It has high frequency dynamic characteristics that are very sensitive to attachment location.

Shift systems also contribute to pass the vibration with the cables that are connected to the transmission until the shift lever. The frequencies involved could generate noise and harshness on the lever. Dampers and terminals designs and durometer need to be dimension to filter the vibration. Clutch pipes also contribute to pass the vibration on the pedal box and depending on the vehicle application, a damper in line is necessary to filter this vibration on the fluid.

Side shafts could contribute to reduce to torsional vibration of the system according to the transference to the transmission by the differential. Increasing the stiffness the torsional vibration is reduced. However to increase the stiffness of the side shaft a bigger diameter is needed, causing sometimes problems of assembly. The graph below is comparing the gain on the vehicle.

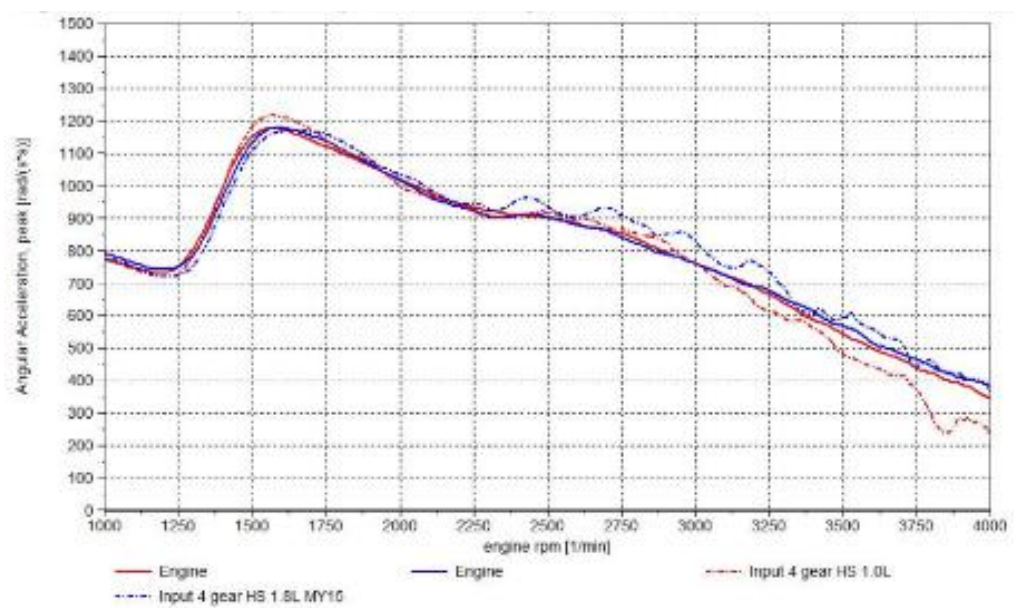

Fig.08: Graph Angular Acceleration vs. engine rpm comparing stiffness of drive shaft. 


\subsection{Air borne energy}

In airborne sound sources, forced or free vibrations are excited in air. The airborne path between engine surfaces and occupant, only attenuated by acoustic isolation such as offered by the firewall, or the airborne path between the wheel/road contact zone and the car interior. The shape, dimensions and materials in this transfer path define the degree of noise reduction (or enhancement, e.g. by acoustic resonance effects).

There are essentially two different treatments for solving noise and vibration problems. Absorbers and barriers are used for airborne noise problems.

An absorber is a product that reduces sound wave reflections from hard surfaces and thereby minimizes sound build-up or reverberation within an enclosed space. Typically, an absorber is either a fibrous or a cellular material that is both porous and elastic. Sound waves enter the material and dissipate to thermal energy via viscous dissipation and/or internal friction. The sound wave interaction between the solid constituent of the material and the air within the material influences the performance of the absorber. A critical physical characteristic that affects the performance of an absorber is flow resistance. The performance of a pore-elastic material can be enhanced using flow-resistive facings. Six Factors impacting performance are: bulk density; solid density; flow resistivity; Young's modulus; shear modulus; thermal length; viscous length; tortuosity; and porosity. Depending on the type of facing and its flow resistance characteristics, the performance of an absorption material could be affected dramatically. Therefore, if you know the benefits and limitations of the facing material, you can use this information to your benefit. Various factors that affect performance of an absorption material are shown qualitatively on the figure below:

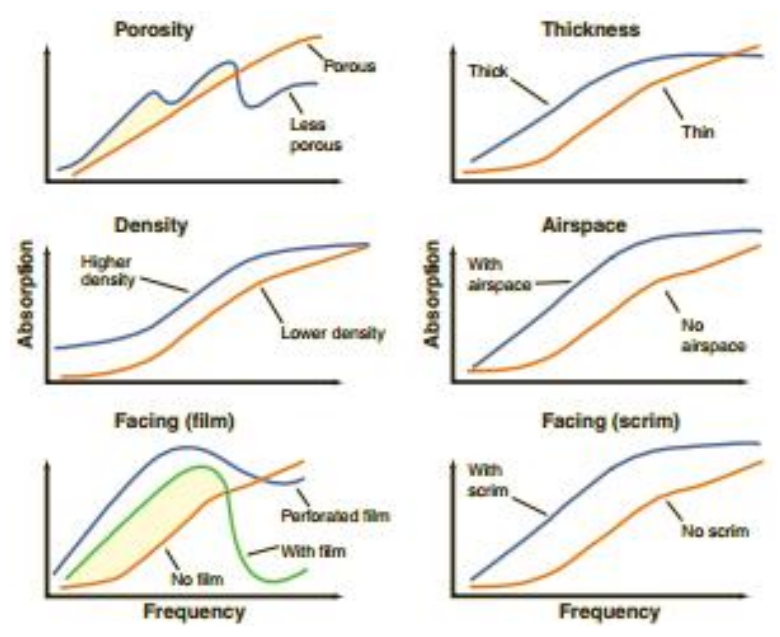

Fig.09: Factor that affects performance of absorption.

A barrier is a material that blocks sound propagating from one area to another, for example, from the engine compartment to inside the vehicle. A barrier is a nonporous, massive, and limp material. If there are holes in the barrier, the sound energy, depending on the wavelength of sound and the size of the hole, has the potential to pass to the other side without attenuation. Therefore, a barrier with a hole cannot effectively block sound with 
one-quarter wavelength equal to or shorter than the size of the hole. The more massive the barrier, the better its ability to block sound. As sound waves strike the barrier material, the mass of the barrier does not allow it to vibrate. Therefore. The air on the other side of the barrier does not get excited, and the sound energy does not propagate easily. A barrier needs to be limp to prevent resonance effects, so it does not re-radiate sound easily.

Dashboard material by itself is a good material for noise control. Unfortunately, that alone is insufficient to control noise propagation from the engine compartment to inside the cabin. Therefore, additional acoustical materials such as dash mats are used to enhance performance. Unfortunately, there are holes and cutouts on the dashboard and dash mat for different accessories to pass through between the engine compartment and cabin. These accessories have the potential to introduce noise leakage paths.

Because of this and in the effort to reduce weight, many vehicles use lightweight dash mat constructions. The dash mats in this case are essentially two different absorption materials with different resistive characteristics that have the potential to enhance the net acoustical performance of the treatment. But for luxury and very high-quality vehicles, barriers are one of the few ways to obtain the acoustical requirements that consumers desire.

Therefore, in all world-class vehicles, regardless of manufacturer, a lot of barrier materials are used to achieve the target acoustics. While using barriers to reduce noise, one needs to be familiar with the performance benefits and limitations of single-wall and multiwall (also known as double wall) constructions. The sound transmission loss of a double-wall construction system depends on various factors:

- Surface density and stiffness of the individual walls.

- Space and material (decoupler material) used between the two walls.

- Stiffness of the decoupler material.

Perhaps the most challenging task of all is ensuring that the best sound package has been developed in terms of its value. The value (not just monetary) in today's global automotive market consists of: cost; weight; durability; functionality, recyclability; packaging space; environmental impact; performance; and ergonomics. One needs to understand in developing the sound package treatment, that significant effort is needed beyond individual material performance to achieve optimum balance of performance for various noise source and operating condition combinations. The performance of a given treatment component may also depend somewhat on the presence of other components. In other words, there may be situations where increasing the performance of a sound package treatment may not have any overall impact on vehicle acoustics. Therefore, it not only becomes difficult to determine the true importance of certain materials, in some cases the acoustical requirements of certain parts may become very minimal. Unfortunately, this would not be known unless a thorough study is done on the source-path-receiver analysis. In addition, the user also needs to make every effort to minimize flanking paths. 

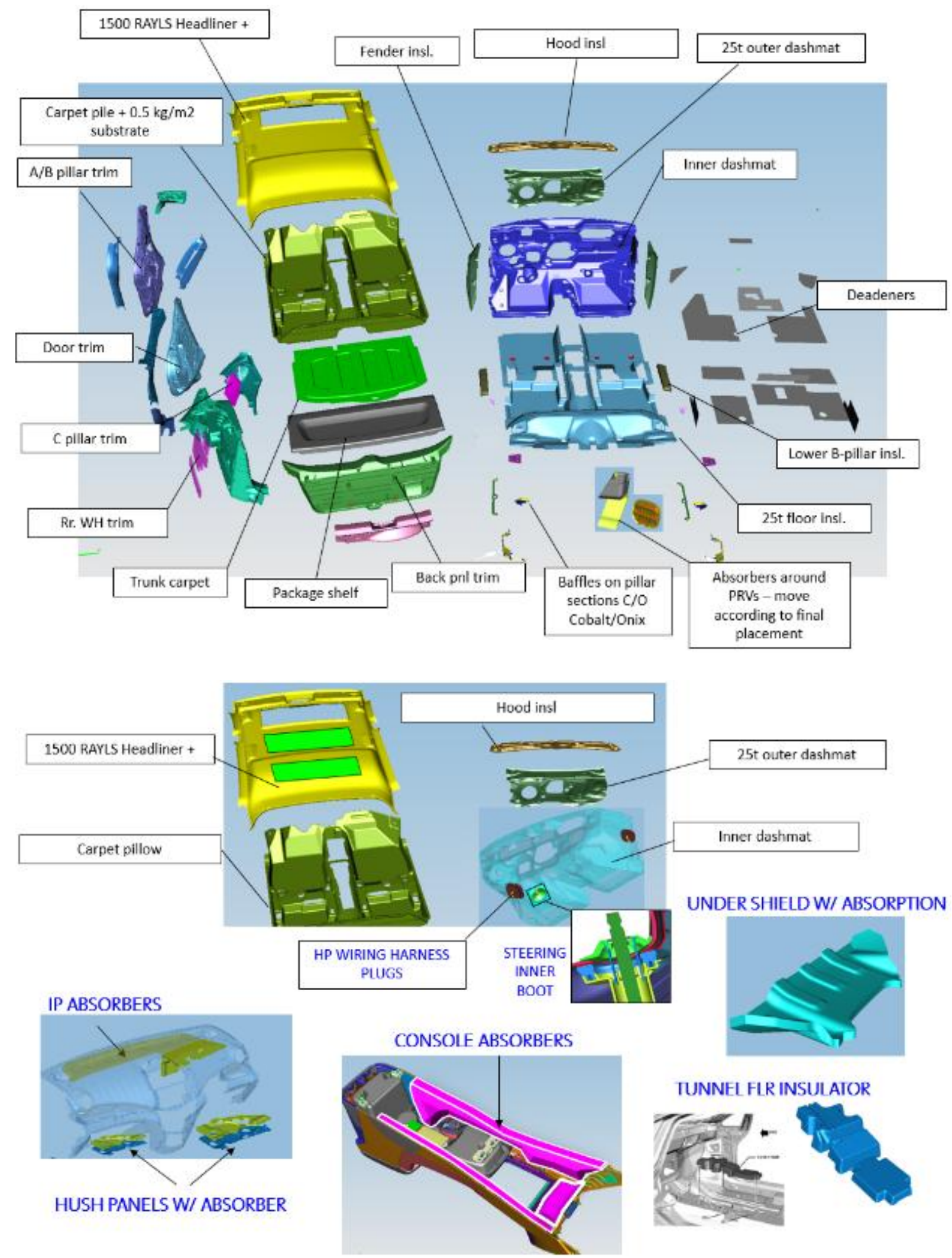

Fig.10: Vehicle insulation. 


\section{Simulations}

Driveline Torsional Analysis for GSV Platform with Fam1 GV4 1.4L (LKJ) F19 to support clutch tuning activities to understand the rattle noise phenomenon.

2.1 Results

The proposals analyzed were:

- Damper stiffness at last stage: $7.7 \mathrm{Nm} /{ }^{\circ}$ and $9.5 \mathrm{Nm} /{ }^{\circ}$

- Damper hysteresis at last stage: $8.7 \sim 12 \mathrm{Nm}$

Additionally, it was analyzed also the sideshaft stiffness impact. Two configurations os sideshafts were used:

- MY15: $\mathrm{LH} 74.2 \mathrm{Nm} /{ }^{\circ}$ and $\mathrm{RH} 58.6 \mathrm{Nm} /{ }^{\circ}$

- $\mathrm{MY} 17: \mathrm{LH} 76.6 \mathrm{Nm} /{ }^{\circ}$ and $\mathrm{RH} 45.1 \mathrm{Nm} /{ }^{\circ}$

\begin{tabular}{|c|c|c|c|c|}
\hline & Test nr. & Min & Nom & $\operatorname{Max}$ \\
\hline Clutch cover & L-01020-0C35-01 & & & \\
\hline Clutch disec & L-03020-1026-01 & EP20150922 & EP20150387 & EP20150918 \\
\hline Hysteresis & & Min & Nom & $\operatorname{Max}$ \\
\hline Hys. Coast [Nm] & & 8.87 & 11.72 & 13.34 \\
\hline Hys. Pre ist [Nm] & & 0.52 & 0.62 & 0.87 \\
\hline Hys. Pre 2nd [Nm] & & 1.37 & 2.03 & 2.72 \\
\hline Hys, Drive [Nm] & & 8.71 & 12.03 & 15 \\
\hline Stt. Coast [Nm/"] & & 4.09 & 4.97 & 4.93 \\
\hline Stf. Pre 1st [Nm/7] & & 0.22 & 0.18 & 0.14 \\
\hline Stf. Pre 2nd [Nm/"] & & 0.77 & 0.88 & 0.97 \\
\hline Stf. Drive $\left[\mathrm{Nm} /{ }^{\prime \prime}\right]$ & & 9.60 & 9.54 & 9.51 \\
\hline
\end{tabular}

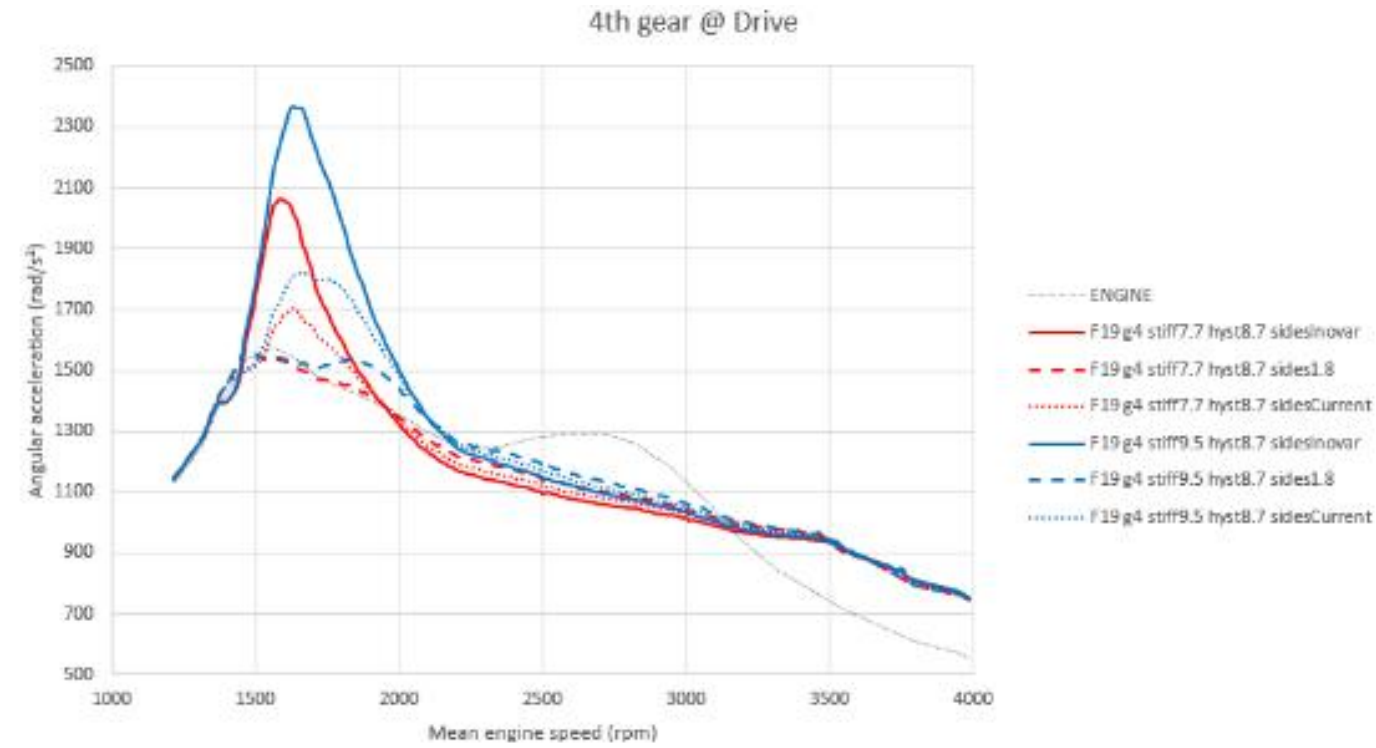

Fig.11: Simulation results 
Based on supplier parameters limitations, the best compromise in terms of angular acceleration of transmission inputshaft is achieved using a low value of damper stiffness at last stage $\left(7.7 \mathrm{Nm} /{ }^{\circ}\right)$ and around $8.7 \mathrm{Nm}$ of hysteresis.

Added to that, using stiffer sideshaft also shows better torsional results.

The model was adapted to use as input the measured engine angular acceleration from Supplier measurements. Backlashes, tolerances and dynamic oil level are not considered on the model.

\section{Vehicle Measurements}

For vehicle measurements was used an accelerometer and microphones to record all datas.
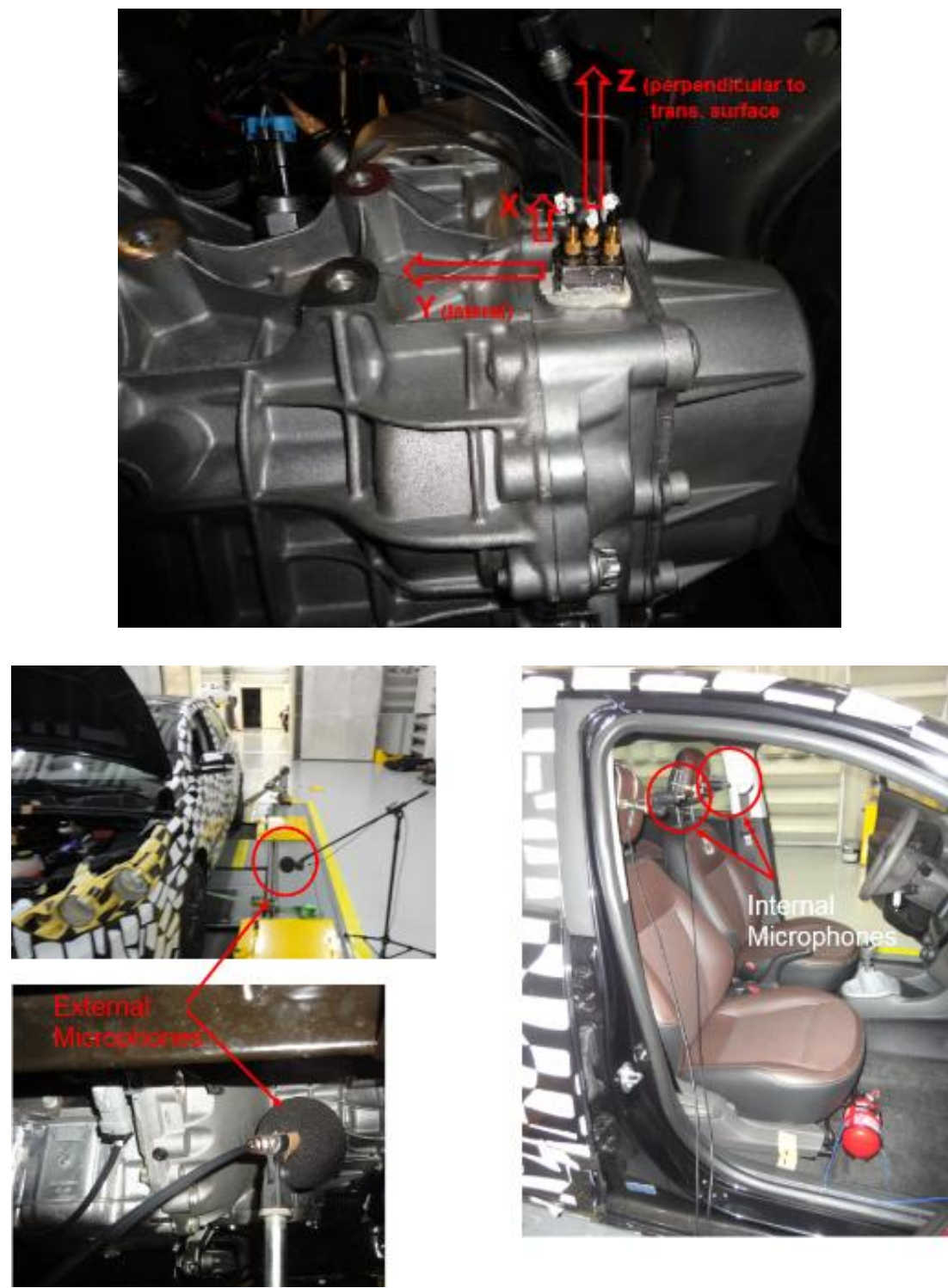

Fig.12: Vehicle instrumentation 


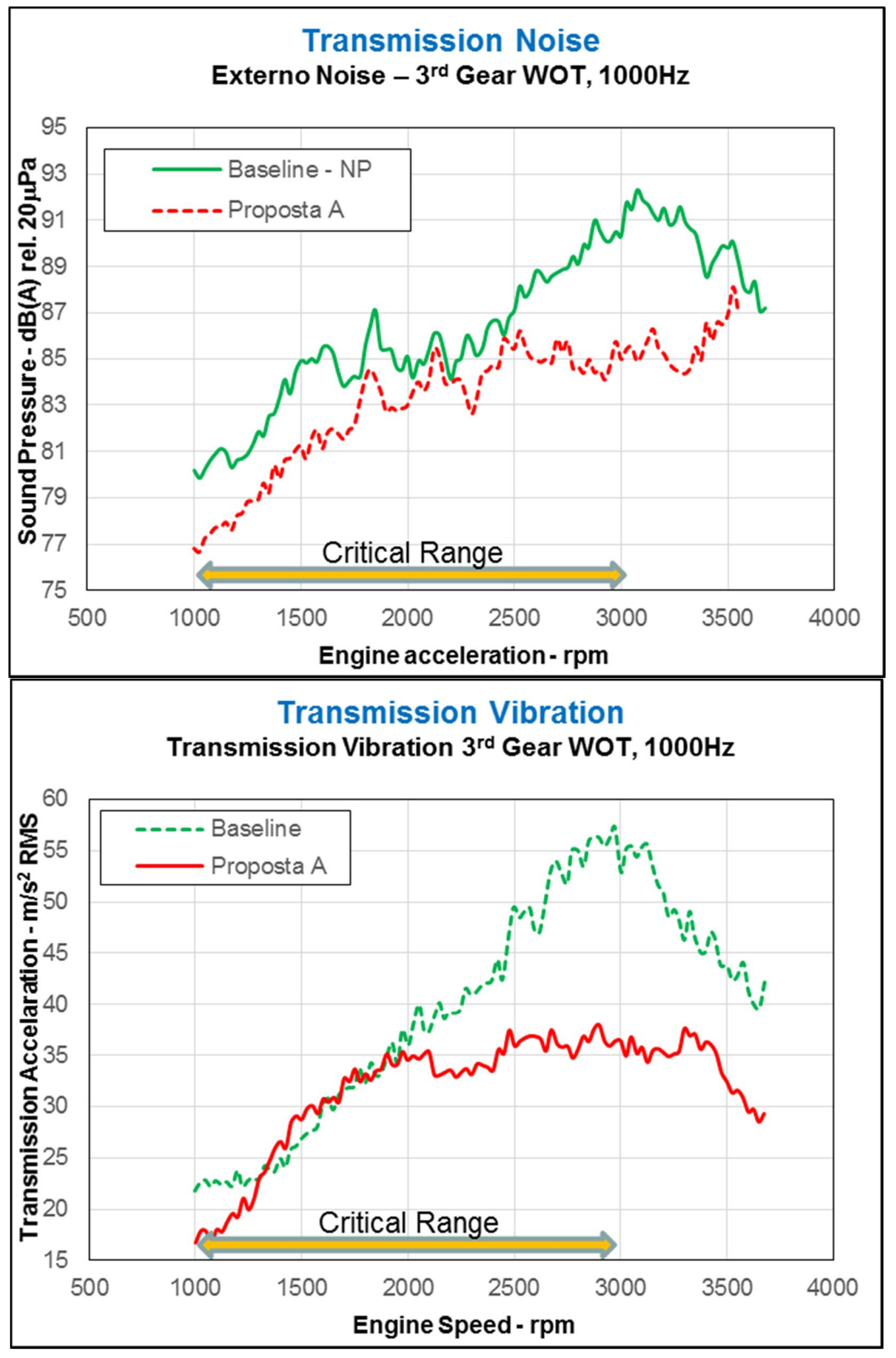

Fig.12: Vehicle measurements 


\section{CONCLUSION}

The cause of rattling noise is torsional vibration of transmission components that are not under load, that move backwards and forwards within their functional clearances. This noise is perceived as distinct from other sources of noise, and is intrusive because of its undesirable character. The transmission parameters backlash, axial clearance and main center distance were varied by experimental analyses in test stand trials, showing the effect on the propensity to rattle. Also the vehicle insulator, stiffness of axial half shaft, clutch disc torsional stiffness and hysteresis, are parameters. We can solve and optimize these parameters, it was possible to minimize the rattling noise. Measures internal to the transmission to reduce loose part vibration in vehicle transmissions were also considered. The effectiveness, in terms of minimizing rattling proneness, of making the better results. All internal transmission measures discerned as effective in reducing rattling and noise need to be examined in terms of service life and possible side effects in all the operating states arising in a vehicle transmission.

\section{REFERENCE}

1. Lechner, G.; Naunheimer, H.: Fahrzeuggetriebe, Springer-Verlag, Berlin, Heidelberg, 1994

2. Weidner, G.: Klappern und Rasseln von Fahrzeuggetrieben, Dissertation, Stuttgart University,1991

3. Lang, C.-H.: Losteilgerusche von Fahrzeuggetrieben, Dissertation, Stuttgart University, 1997

4. Dogan, S. N., Ryborz, J., Lechner, G.: Simulation von Losteilschwingungen in Fahrzeuggetrieben - Vergleich Messung mit Simulation, Antriebstechnik 37 (1998) Nr. 7

5. Dogan, S. N., Lechner, G.: Maßnahmen zur Verringerung von Losteilschwingungen in Fahrzeuggetrieben, ATZ Automobiltechnische Zeitschrift 100 (1998) 10 